\section{SAFETY ADVANTAGES OF PRESTRESSED CONCRETE REACTOR VESSELS}

ZDENĚK P. BAŽANT Northwestern University

Technological Institute, Department of Civil Engineering Evanston, Illinois 60201

Received December 12, 1975

Accepted for Publication March 12, 1976
REACTORS

KEYWORDS: power reactors, relisbility, pressure vessels, prestressed concrete, safety, failures, comparative evaluations, stresses
An attempt has been made to explain the advantages of the behavior of prestressed concrete reactor vessels in a simple, brief, and qualitative form. In contrast to the property of spontaneous propagation of brittle fracture in massive steel plates, the failure of one prestressing wire or tendon does not propagate into the adjacent wires or tendons. The pressure-deflection curve does not end by a sudden failure, but the decrease of slope on approach to failure is gradual and even after formation of through cracks the vessel would close if depressurized. The energy absorption capability in post-elastic deformation is much higher than that of a steel vessel which could fail by brittle fracture. A weak part is the top closure slab, but if it is designed sufficiently thick to assure that a separation of a conical segment does not create a hole through the slab, and if much higher safety factors are used than those for the barrel sections, a very favorable failure behavior is assured. Comparison of the behavior of concrete and steel vessels in accidental exposure to high temperature and the role of moisture in concrete deserve further investigation.

\section{INTRODUCTION}

Designers of prestressed concrete reactor vessels (PCRVs) often claim that these vessels are safer than the steel vessels. Although the steel vessels cannot be said to be unsafe, these claims are correct, as far as failure due to internal pressure alone is concerned. However, the reasons have not been clearly stated yet, and the case has not been made in a convincing form. An attempt to do so is made in the brief and qualitative analysis that follows.

\section{PROPAGATION OF LOCAL FAILURE}

When the internal pressure in a PCRV is increased beyond the maximum design pressure, concrete undergoes gradual tensile cracking until continuous through cracks are formed. ${ }^{1-5}$ However, these cracks do not represent failure, for their opening is small and does not cause rupture of the steel liner, and the tensile load in the cracked cross section is carried by the prestressing tendons as well as the mild steel reinforcement of concrete. The internal pressure can be further considerably increased before the vessel fails. In the final stage, in which the concrete wall is cracked through, the integrity of the vessel depends primarily on the system of parallel prestressing tendons (Fig. 1a), each consisting of a number of parallel wires, and also on the mesh of unprestressed reinforcement in concrete. This system has the important property such that a failure of an element, e.g., one wire or one whole tendon, has a negligible effect on the stress in the remaining elements; e.g., if one out of 100 tendons fails, the stress in all the remaining tendons is increased only by $\sim 1 \%$. This property is a general characteristic of composites in which strong continuous elements (tendons) are embedded in a relatively weak matrix (concrete).

A steel vessel can fail either by plastic yielding or by brittle fracture. The plastic failure has far better safety characteristics, but the possibility of brittle fracture cannot be excluded. The brittle fracture is a failure mode that originates from a critical flaw or crack in the wall of the vessel. The sharp edges of the crack cause severe stress concentration ( $A$ in Fig. 1a). When the stress concentration exceeds a certain level, as indicated by the stress intensity factor, ${ }^{6}$ which is a measure of the energy release rate at crack extension, the crack propagates into an adjacent material element ( $B$ in Fig. 1b). By contrast to the system of parallel independent elements, this 
SAFETY ADVANTAGES OF PRESTRESSED CONCRETE REACTOR VESSELS

Zdeněk $\mathrm{P}$. Bažant ( $\mathrm{PhD}$, Czechoslovak Academy of Sciences, 1963) is a professor at Northwestern University, Evanston, Illinois. Under the sponsorship of the National Science Foundation, the U.S. Energy Research and Development Administration, and the Advanced Research Projects Agency, he has been directing an extensive research program in structural mechanics problems of nuclear concrete structures.

Zdeněk P. Bažant

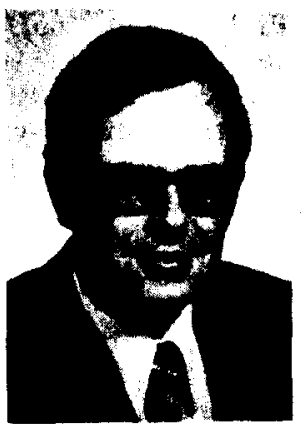

SEPTEMBER 1976 

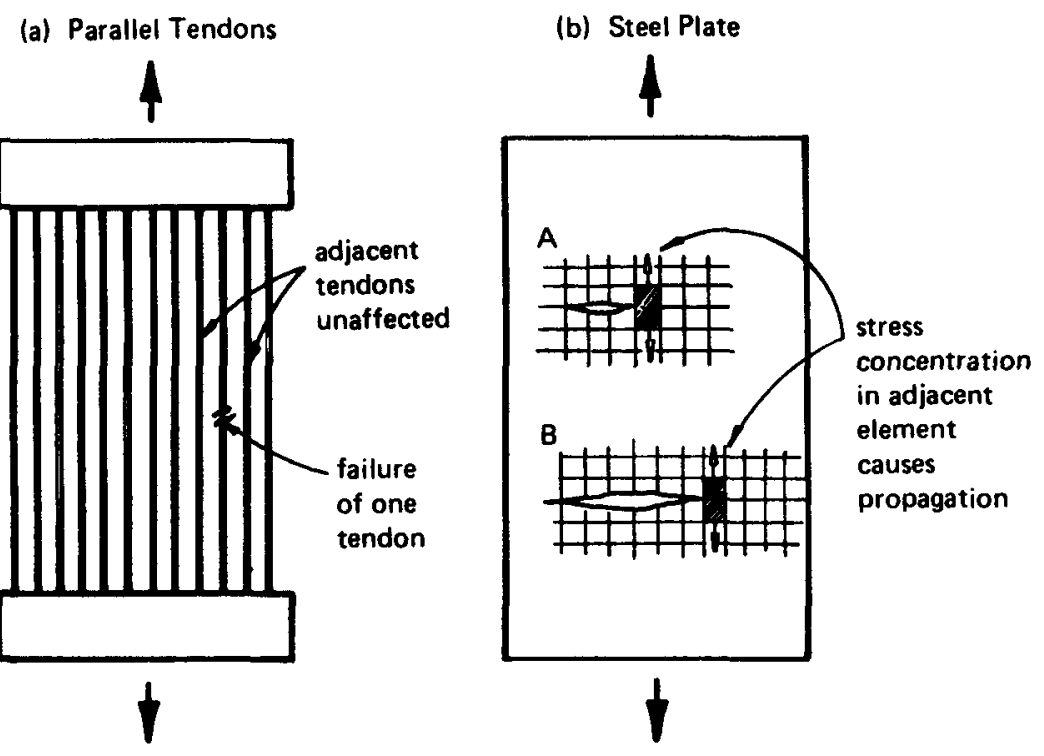

(c) Reinforced Concrete

Fig. 1. Schematic diagram visualizing the propagation of failure in various structural systems.

results in a large stress concentration in the next material element, which now appears just ahead of the crack edge. Thus, the crack propagates into the next material element ( $B$ in Fig. 1b), and the cycle just described is repeated. The process of fracture, once started, can propagate spontaneously and very rapidly until a large crack is formed. The propagation occurs even when no work is done on the boundary of the steel plate, for unloading of elastic strain energy from the whole structure into the crack tip may suffice to drive the crack.

The for egoing process of brittle fracture contrasts with the failure of a system of independent parallel tendons in which the failure of one element (tendon) cannot cause spontaneous propagation of the fallure through all elements of the system. It is safer to depend on a number of cross sections of independent parallel carrying elements (tendons, wires, and reinforcing bars) than it is to depend on a single, large, monolitic cross section of one carrying element (steel plate). This conclusion is corroborated by the fact that the statistical distribution of failure loads is much narrower for the former of the preceding two cases. This is true regardless of the fact that prestressing wires can be produced with greater homogeneity than massive steel plates.

The absence of self-propagating failure is also characteristic of the tensile cracking of densely reinforced concrete, which precedes the formation of through cracks in the wall. This is due to the fact that a sufficiently dense mesh of reinforce- ments delays the coalescence of a system of tensile microcracks into one large crack. Furthermore, even when a large crack is formed, its propagation requires considerable increase of stress because the stress concentration ahead of the crack front is strongly reduced by a large and densely microcracked zone, and because the formation of the microcracked zone consumes much more energy than the formation of the main crack itself. Thus, the process of tensile fallure of reinforced concrete is closer to the case of parallel independent elements rather than the case of brittle fracture. In addition, the individual nonprestressed reinforcing bars embedded in concrete are much less likely to exhibit brittle failure than is a massive steel plate of the reactor vessel. Rather, most of the bars are certain to extend plastically, with a long yield plateau.

\section{LOAD-DEFLECTION DIAGRAM AND ENERGY ABSORPTION}

The differences between brittle failure of the steel vessels and gradua: failure of the PCRVs can be clarified further by considering the pressure-deflection diagrams, which are exemplified for both cases in Fig. 2. The failure point on these diagrams is the maximum point, $m$. From the theory of structural stability, it is known that equilibrium corresponding to the segments of negative slope (dashed lines in Figs. $2 a$ and $2 b$ ) is unstable; any state on such a segment is transient and deflection continues spontaneously. 
(a) Concrete Vessel

(Barrel Failure)

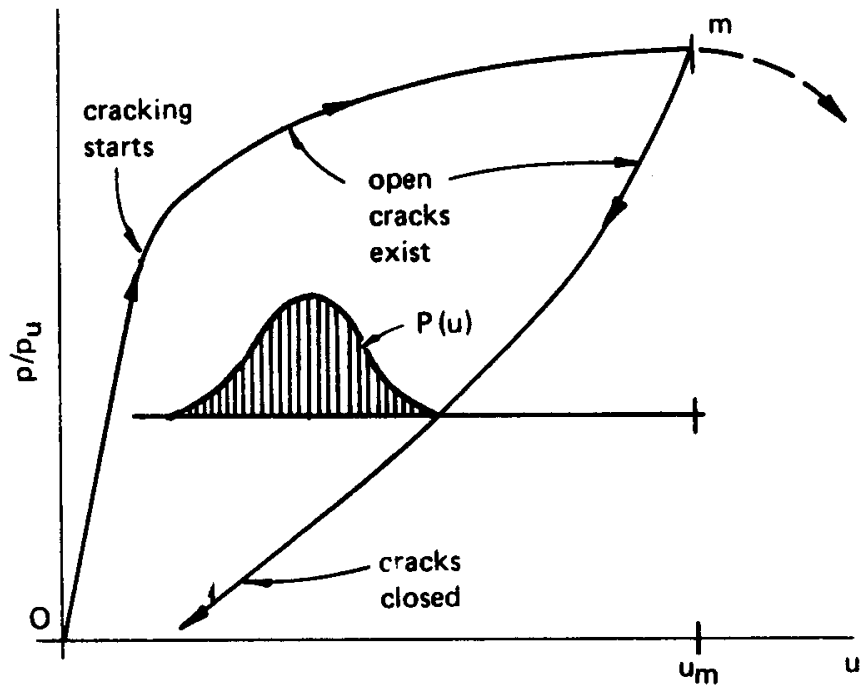

(b) Steel Vessel

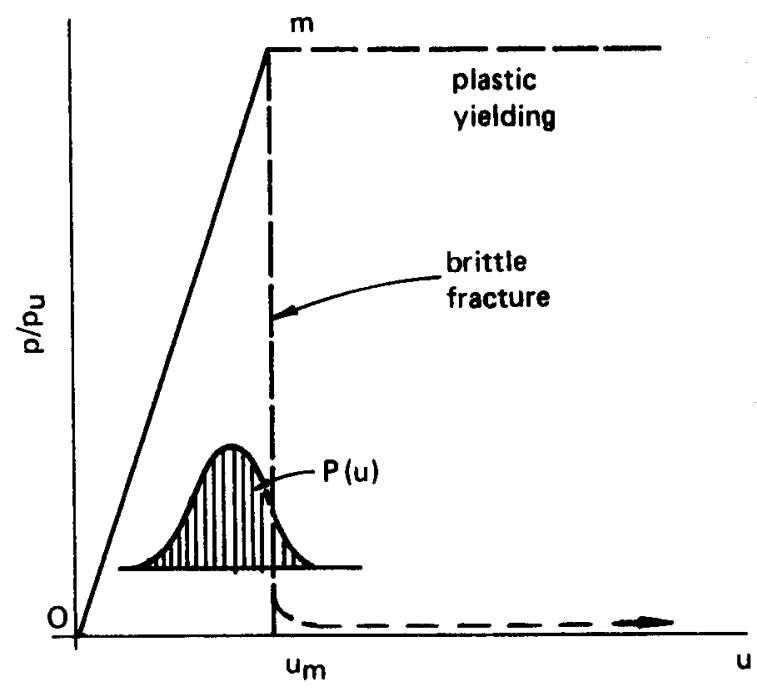

deflection

Fig. 2. Comparison of pressure-deflection diagrams of concrete and steel vessels.

In a concrete vessel, the cracks develop and open gradually (Fig. 2a), which results in a gradual decrease of stiffness, manifested as a decrease of slope of the failure diagram. Before reaching the maximum point (failure point), the gradual appearance of large cracks gives a warning that the failure is imminent. If the vessel is depressurized before reaching the maximum point, the prestressing tendons cause the cracks to close and the integrity of the vessel is regained. Crack closing is achieved even though the deformation is not quite reversible and the prestress across the closed cracks after depressurization is less than the original prestress before pressurization. The cracks begin closing only after the internal pressure drops well below the value at which the cracks formed. These irreversibilities are caused mainly by plastic strains in reinforcing bars, tendons, and steel liners during overpressure (hysteresis).

In a steel vessel, on the other hand, no significant cracking takes place before the final, sudden collapse (Fig. 2b). If a crack appears, the vessel can never return to the original shape and regain integrity.

From the statistical viewpoint, the probability density distribution curves of the deflections can be considered [see $P(u)$ in Fig. 2]. Obviously, due to the gradual decrease of slope of the pressuredeflection diagram, in a concrete vessel the probability $P(u)$ of exceeding deflection, $u_{m}$, for the maximum point is considerably less.

Due to the fact that cracks appear in a concrete vessel before the final failure, the leak tightness is not lost suddenly but gradually. Part of the gas from the vessel may leak into the containment before the total failure; this reduces the interior pressure and the vessel subsequently closes. On the other hand, in a steel vessel failing by brittle fracture, the failure, if any, is always permanent and total.

It is interesting to note that the differences in the failure process of concrete and steel vessels are to some degree analogous to the differences between buckling failures of compressed thin plates and axially compressed thin cylinorical shells. Here, too, one of these structures exhibits a gradual decrease of slope of the deflection curve and large deformations (Curve a, Fig. 3), while the other suddenly unloads at a relatively small

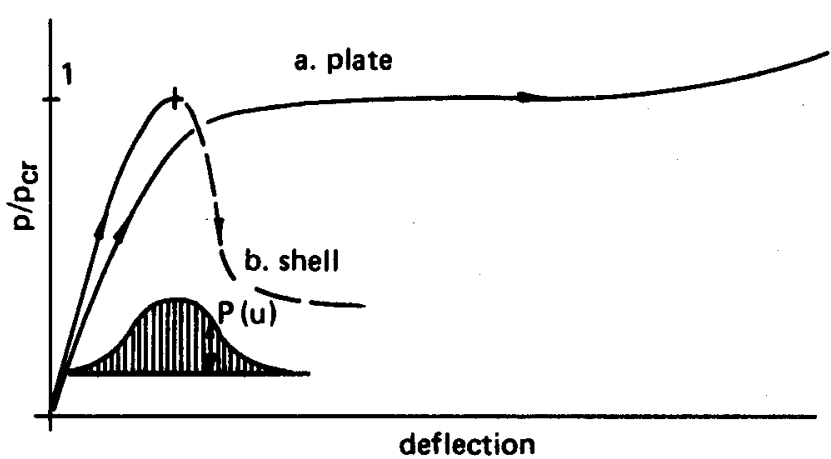

Fig. 3. Load-deflection diagrams of plates and shells shown for the purpose of analogy. 
deformation (shell, Curve b, Fig. 3). This fact, resulting from inevitable initial imperfections of shells, has not been theoretically understood until about 1940, although it has been known from experiments since about 1910 that the compressed cylindrical shells are less safe as structural elements and must be designed with much higher safety margins. ${ }^{7}$

Another property important for structural safety is the energy absorption capability. The energy that is absorbed by the vessel before reaching the peak pressure, point $m$, is equal to the area under the load-deflection curve; i.e., area $O m u_{m}$ in Fig. 2a or 2b. It is seen from this figure that, in case of brittle fracture, the energy absorption of a steel vessel is much smaller than that of a PCRV designed for the same maximum pressure. Highenergy adsorption is particularly important for resisting a sudden high-pressure pulse in a hypothetical core disruptive accident. Likewise, a concrete vessel has a high-energy adsorption capability when subjected to an external blast or missile impact.

The large inertial mass of concrete vessel as compared with steel vessel is also advantageous for resisting the impact loads. Regarding earthquakes, PCRVs are fail-proof without any special design measures, the foundation excepted.

\section{STRUCTURAL MODES OF FAILURE}

For the sake of simplicity, consider a singlecavity cylindrical vessel. There are two different modes in which such a vessel can fail: (a) the barrel failure, Fig. 4a, and (b) the top slab failure, Fig. 4b. All preceding discussions refer to the case of barrel failure. The top slab failure has rather different characteristics and can fail in an almost brittle fashion, characterized by a relatively rapid drop of pressure-deflection diagram without much prior cracking.

The failure of the top slab usually initiates by tensile cracks of 45-deg inclination, called diagonal shear cracks. These cracks later extend to form a cone, Fig. 4b, that may become loose in an explosive manner. The top slab failure entirely depends on the tensile failure behavior of concrete with its reinforcing mesh, unaided by the favorable failure characteristics of the system of parallel prestressing tendons. It is possible to design the top closure in the form of a prestressed, thick, hemispherical dome. The dome has the same favorable failure characteristics as the barrel section. This has been done, but appears to be extremely inconvenient in construction as well as in service. Therefore, the PCRVs are designed in a way that makes the barrel fail at (a) Barrel Failure

(b) Top Slab Failure
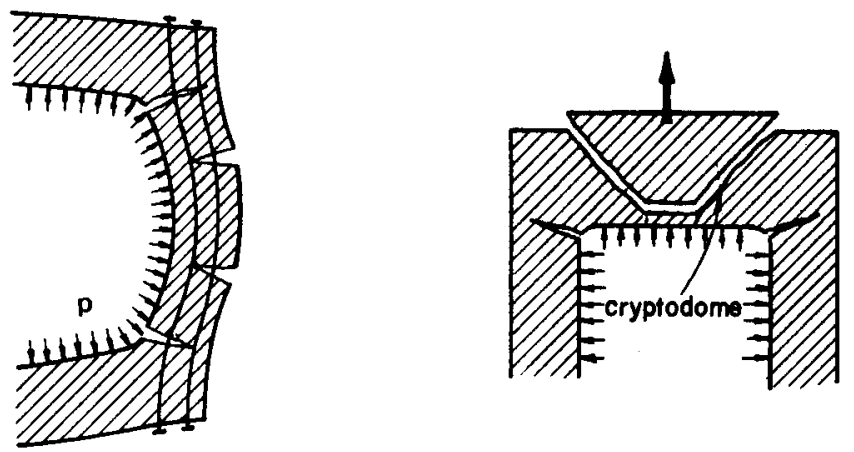

Fig. 4. Structural failure modes of a single-cavity PCRV. (The barrel failure is accompanied by large vertical radial cracks distributed along the circumference.)

a pressure that is much smaller than the pressure that would make the top slab fail. This is achieved by using much higher safety factors for the top slab than for the barrel section, 3 to 4 instead of 2. Furthermore, the top slab is designed sufficiently thick so as to fail in a mode that is less than catastrophic. Namely, when the top slab is made very thick, the loosening of a conical segment does not produce a hole all the way through the slab, but leaves continuous concrete in the shape of a cryptodome, Fig. 4, so that a leak does not occur. The leak is obtained only after further increase of pressure leading to a shear punching failure of the thin central part of the remaining cryptodome.

Another sensitive point is the fact that the bending moment at discontinuities and large cracks can be accompanied by a large transverse shear force. However, with an adequate mild steel reinforcement, the transmission of shear force can be assured.

The structural failure characteristics of the multicavity PCRVs are similar, but their discussion would be too complicated for our purposes.

\section{OTHER ASPECTS}

It should be mentioned that the primary loadbearing elements, the tendons, can be installed in such a manner that their inspection and eventual replacement is possible at any time during service life. The homogeneity of prestressing wires is better and the detection of flaws is easier than in massive steel plates. All load-bearing structural steel, which does not include the steel liner, is shielded by concrete from radiation. Furthermore, this steel does not suffer from plastic thermal cycling during temperature changes in the 
reactor cavity (at shutdowns), and also, the steel liner in a gas-cooled reactor can be effectively thermally insulated to prevent thermal cycling. This eliminates the main possible sources of material damage.

In a gas-cooled reactor, a very significant improvement of safety arises from the fact that the complete primary loop (reactor core, coolant ctrculators, and steam generators) can be contained within the vessel, thus reducing the consequences of possible piping failures.

\section{CONGLUSION}

From the preceding considerations it follows that in failure due to internal pressure, a PCRV has more favorable characteristics than a steel vessel. Hence, a PCRV is safer than a steel vessel in this basic type of loading.

However, this conclusion only concerns the theoretical safety margins and, especially, does not mean that steel vessels are unsafe. The number of reactor hours of experience accumulated with steel vessels for water-cooled reactors is much higher than that accumulated with PCRVs for gas-cooled reactors, and no failure of significant consequence has been recorded thus far.

The present analysis has not taken into account the question of the differences in the probability of a catastrophic increase of internal pressure in various types of reactors. In a complete analysis, this would have to be considered as well.

The preceding analysis is restricted to the failure due to internal pressure and does not apply to cases with significant increase of temperature in concrete, which can theoretically occur in certain hypothetical accidents of extremely low probability. The PCRVs are probably more sensitive to temperature increase than are steel vessels, provided the temperature is not so high as to cause an excessive creep rate in the steel vessel. On the other hand, concrete vessels can better accommodate a localized internal distress due to temperature increase. Aside from that, the interior of the wall takes quite long to heat up, and cooling coils can be embedded in concrete under the steel liner to reduce accidental temperature ,rise. Consideration of the questions just mentioned $^{8}$ will require extensive research. The question of whether the probability of high tem- perature ever penetrating through the bulk of the concrete wall is of any significant value has not been settled.

Creep and moisture migration effects have not been considered in the present analysis. These effects are normally not of major importance for failure due to internal pressure, although they must be considered if the desired safety margins should be maintained, if the design should be economical, and if serviceability should not be endangered by excessive cracking. ${ }^{8}$ The presence of moisture in concrete is undoubtedly detrimental, to some extent, when concrete is heated to a high temperature or is exposed to liquid sodium; but further research will probably allow these effects to be minimized.

\section{ACKNOWLEDGMENT}

This paper has been written in connection with research supported by the National Science Foundation under Grants GK-26030 and ENG75-14848.

\section{REFERENCES}

1. Proc. 1st, 2nd, and 3rd Int. Conf. Structural Mechanics in Reactor Technology, Berlin (1971 and 1973), London (1975), Commission of European Communities, Brussels; also, Proc. PCR V Conf., York, U.K., (Sep. 1975).

2. "Concrete for Nuclear Reactors," Proc. ACI Int. Seminar, West Berlin, Oct. 1970, ACI SP-34, American Concrete Institute, Detroit (1972).

3. Standard Code for Concrete Reactor Vessels and Containments, American Society of Mechanical Engineers' Boiler and Pressure Vessel Code, Sec. CB, Concrete Reactor Vessels, and American Concrete Institute Standard 359, New York (1974).

4. J. L. PLAEHN, "Practical Arproach to the Design Philosophy of PCRV," Proc. ACI Int. Seminar, West Berlin, ACI SP-34, pp. 235-266, American Concrete Institute, Detroit (1972).

5. C. O. PEINADO, J. L. PLAEHN, and M. J. WAY, "Fort St. Vrain PCRV," ASCE Meeting Preprint 1068, American Society of Civil Engineers (Oct. 1969).

6. J. F. KNOTT, Fundamentals of Fracture Mechanics, John Wiley \& Sons, Inc., New York (1973).

7. A. CHAJES, Principles of Structural Stability Theory, Prentice-Hall, Inc., Englewood Cliffs, New Jersey (1974).

8. Z. P. BAŽANT, "Some Qu stions of Material Inelasticity and Failure in the Design of Concrete Structures for Nuclear Reactors," Proc. 3rd Int. Conf. Structural Mechanics in Reactor Technology. Vol. 3, Paper H1/1, Commission of European Communities, Brussels (1975). 